\title{
Clinical profile of ocular toxoplasmosis in the Universiti Sains Malaysia Hospital - 7-year review
}

\author{
TONG JONG HAW MATTHEW ${ }^{A-F}$, EMBONG ZUNAINA ${ }^{A, B, D, E}$, ADIL HUSSEIN ${ }^{B}$, AHMAD TAJUDIN \\ LIZA-SHARMINI ${ }^{B}$, WAN HITAM WAN-HAZABBAH', ISMAIL SHATRIAH ${ }^{B}$
}

Department of Ophthalmology, School of Medical Sciences, Universiti Sains Malaysia, Kubang Kerian, Kelantan, Malaysia

A - Study Design, B - Data Collection, C - Statistical Analysis, D - Data Interpretation, E - Manuscript Preparation, F - Literature Search, G - Funds Collection

\begin{abstract}
Summary Background. Toxoplasmosis is caused by infection from a ubiquitous obligate intracellular parasite known as Toxoplasma gondii, which can infect both human and warm-blooded animals. This infection is commonly prevalent in developing country. Objectives. The purpose of this study is to report on a 7-year review of ocular toxoplasmosis patients that presented to the Universiti Sains Malaysia Hospital.

Material and methods. This is a retrospective review of cases. The demographic data, ocular manifestations, clinical and laboratory profiles, treatment and disease outcome were collected from the patients' medical records from January 2010 to December 2016. Results. A total of 16 patients with a diagnosis of ocular toxoplasmosis were identified. The mean age was 43.2 , with standard deviation \pm 15.4 years old. The percentage was $62.5 \%$ (10 patients) females and $37.5 \%$ (6 patients) male. The majority of patients were Malay (93.4\%, 15 patients). The most common classification of uveitis was panuveitis (56.3\%, 9 patients), followed by posterior uveitis ( $25 \%, 4$ patients), anterior uveitis (12.5\%, 2 patients) and intermediate uveitis (6.2\%, 1 patient). Keratic precipitate ( $75 \%, 12$ patients) and vitritis (75\%, 12 patients) were the most common ocular manifestation of ocular toxoplasmosis. A serological test for toxoplasmosis found only 1 patient with positive serum immunoglobulin $\mathrm{M}$, while all patients displayed non-reactive serum immunoglobulin G (except 1 missing data). All ocular toxoplasmosis patients were treated with oral Azithromycin $500 \mathrm{mg}$ daily for 6 weeks, $81.3 \%$ ( 13 patients) were given an instillation of a steroid eye drop, while $25 \%$ (4 patients) required administration of an oral steroid. All of the patients responded well to treatment, but $25 \%$ ( 4 patients) suffered from recurrence, which required a second course of treatment. Conclusions. Ocular toxoplasmosis is a serious and depilating disease that can cause serious ocular morbidities. This review shows a diverse presentation of ocular toxoplasmosis as a result of toxoplasmosis infection.

Key words: toxoplasmosis, uveitis, retinitis, vitritis.
\end{abstract}

Matthew TJH, Zunaina E, Hussein A, Liza-Sharmini AT, Wan-Hazabbah WH, Shatriah I. Clinical profile of ocular toxoplasmosis in the Universiti Sains Malaysia Hospital - 7-year review. Fam Med Prim Care Rev 2018; 20(1): 13-16, doi: https://doi.org/10.5114/ fmpcr.2018.73698.

\section{Background}

Toxoplasmosis is caused by infection from a ubiquitous obligate intracellular parasite known as Toxoplasma gondii (T. gondii). The parasite can infect both human (intermediate host) and warm-blooded animals, such as felines (definitive host) [1]. The parasite was first described in 1908-1909 by Nicolle and Manceaux at the Pasteur Institute in Tunis, Tunisia, with the first case of ocular toxoplasmosis recorded in 1923 [2]. This infection is commonly prevalent in developing country and parts of Southeast Asia, with approximately one-third of the total human population worldwide being chronically infected [1]. Ocular toxoplasmosis occurs in $2-18 \%$ of patients whom are seropositive [2].

Malaysia is one of the countries endemic for toxoplasmosis infection in the Southeast Asia region. In the context of the local community, seroprevalence of $T$. gondii infection was estimated at $37 \%$ based on anti-toxoplasmosis antibodies [3, 4]. Toxoplasmosis infection can be easily spread through unclean water, unhygienic practices and by consuming undercooked infected meat [5-7]. Moreover, the abundance of felines, as Malaysian's favorite household pet, also contributes to the ease of spread.

Toxoplasmosis infection has caused significant morbidity and mortalities. After acquiring the parasite, only $10-20 \%$ of immunocompetent adults are symptomatic. Symptoms include cervical lymphadenopathy, which may be accompanied by non-specific signs, such as fever, night sweats, malaise, myalgia, rash, abdominal pain and hepatosplenomegaly. Rarely, toxoplasmosis may result in severe and fatal manifestations, such as myocarditis, myositis, pneumonitis or encephalitis [8]. The diagnosis of ocular toxoplasmosis is based on clinical tests with the support of immunological markers.

Ocular inflammation, or uveitis, is the clinical manifestation of ocular toxoplasmosis. Classification of patients with ocular inflammation is based on the International Uveitis Study Group (IUSG) by dividing anatomically into anterior, intermediate, posterior and panuveitis [9]. The severity of anterior chamber reaction can be variable, from quiet to severe inflammation, and can also present as both granulomatous or non-granulomatous inflammation. Patients may also present with cataract as a complication of retinochoroiditis or following severe iridocyclitis. Posterior pole manifestations of the appearance of "headlight in the fog" is pathognomonic, due to a combination of dense vitritis and focal necrotizing retinitis [2]. Severe vitritis may lead to the development of an epiretinal membrane, as well as vitreoretinal tractions to the adjacent area. In typical ocular toxoplasmosis, active lesions are seen as whitish foci of retinochoroiditis adjacent to an area of pigmentation or atrophic scar. An active lesion will eventually result in an atrophic retinochoroidal scar, which healed from the periphery to the center of the lesion. Possible complications of ocular toxoplasmosis include band 
keratopathy, cataract formation, chronic iridocyclitis, secondary glaucoma, cystoid macular edema, retinal detachment and optic atrophy secondary to optic involvement [1].

Diagnosis of ocular toxoplasmosis is mainly based on the clinical examination. However, when in doubt, laboratory tools can help to establish a definite diagnosis. Toxoplasmosis serum immunoglobulin M (IgM) and serum immunoglobulin G (IgG) are quick and easy tests, which have a 60-90\% predictive value [3]. A negative toxoplasmosis immunoglobulin would safely rule out toxoplasmosis infection [2]. Other modalities of tests include enzyme-linked immunosorbent assay (ELISA) and ocular fluid sampling for T. gondii deoxyribonucleic acid (DNA) by polymerase chain reaction (PCR) analysis $[2,5]$.

Treatment of ocular toxoplasmosis includes various combinations of antimicrobial drugs with or without the use of a corticosteroid. The "classic" combinations include pyrimethamine, sulfadiazine and corticosteroid and are the usual combination for treatment of ocular toxoplasmosis. Side effects may include hematological complications, such as thrombocytopenia and leucopenia, despite folinic acid supplements. Other forms of combinations may include Trimethoprim-sulfamethoxazole (Bactrim) or pyrimethamine and azithromycin [5].

\section{Objectives}

The aim of this study is to review the ocular manifestations with clinical and laboratory profiles of ocular toxoplasmosis in the Universiti Sains Malaysia Hospital.

\section{Material and methods}

This was a retrospective record review of ocular toxoplasmosis patients that were registered at the Ophthalmology Clinic of the Universiti Sains Malaysia Hospital, Malaysia, from January 2010 to December 2016. The demographic data, ocular manifestations, clinical and laboratory profiles, treatment and disease outcome were collected from the patients' medical records. The collected data was analyzed using the International Business Machines Corp Statistical Package for the Social Sciences (IBM SPSS) Statistics Version 22. The age distribution was analyzed using the Shapiro-Wilk test and was found to be normal. Data with numerical variables was described as mean and standard deviation, while categorical data was expressed by frequency $(n)$ and percentage. This review of case series was conducted in accordance with the Declaration of Helsinki for human research. The patients' personal identification and clinical data were kept confidential, and the data was reported as collective information.

This study was performed with the consent of the university's ethical committee and was in line with the Declaration of Helsinki.

\section{Results}

There were 16 ocular toxoplasmosis patients registered in the Ophthalmology Clinic of the Universiti Sains Malaysia Hospital from January 2010 to December 2016.

The demographic data of the patients at the time of diagnosis is illustrated in Table 1 . The mean age was 43.2 with standard deviation \pm 15.4 years old. The largest group of patients was in the age range of $21-50$ years old, which made up $50 \%$ (8 patients) of the patient volume. Based on gender, female patients made up of $62.5 \%$ (10 patients) and 7 of them were of childbearing age (18-45 years old). One of the patients was pregnant at 30 weeks of gestation during her presentation. She received treatment and was followed up near to term, but unfortunately, she did not return for a follow up of, and as such, a screening for congenital toxoplasmosis was not done. The majority of the patients were Malay (93.8\%, 15 patients) with only 1 Chinese patient $(6.2 \%)$.

\begin{tabular}{|c|c|c|c|}
\hline Variables & & $\begin{array}{l}\text { Frequency } \\
\text { (n) }\end{array}$ & $\begin{array}{l}\text { Percentage } \\
(\%)\end{array}$ \\
\hline \multicolumn{4}{|l|}{ Age (year) } \\
\hline Mean \pm SD & $43.2 \pm 15.4$ & & \\
\hline \multicolumn{4}{|l|}{ Age group } \\
\hline$<10$ & & 0 & 0 \\
\hline $11-20$ & & 2 & 12.5 \\
\hline $21-50$ & & 8 & 50.0 \\
\hline$>51$ & & 6 & 37.5 \\
\hline \multicolumn{4}{|l|}{ Gender } \\
\hline Male & & 6 & 37.5 \\
\hline Female & & 10 & 62.5 \\
\hline $\begin{array}{l}\text { Childbearing age } \\
\text { (18-45 years old) }\end{array}$ & & 7 & 43.8 \\
\hline $\begin{array}{l}\text { More than } 45 \\
\text { years old }\end{array}$ & & 3 & 18.7 \\
\hline \multicolumn{4}{|l|}{ Race } \\
\hline Malay & & 15 & 93.8 \\
\hline Chinese & & 1 & 6.2 \\
\hline
\end{tabular}

Based on the anatomical classification of uveitis, most of the patients presented with panuveitis ( $56.3 \%$, 9 patients), followed by posterior uveitis ( $25 \%, 4$ patients), anterior uveitis $(12.5 \%$, 2 patients) and intermediate uveitis (6.2\%, 1 patient) (Table 2$)$.

\begin{tabular}{|c|c|c|}
\hline Anatomical classification & Frequency (n) & Percentage (\%) \\
\hline Anterior uveitis & 2 & 12.5 \\
\hline Intermediate uveitis & 1 & 6.2 \\
\hline Posterior uveitis & 4 & 25.0 \\
\hline Panuveitis & 9 & 56.3 \\
\hline
\end{tabular}

The clinical profile of ocular toxoplasmosis is summarized in Table 3. A majority of the patients presented with symptoms involving the right eye $(56.3 \%$, 9 patients), of which $75 \%$ (12 patients) complained of blurry of vision, followed by floaters ( $25 \%$, 4 patients), eye redness ( $25 \%, 4$ patients) and eye pain ( $18.8 \%$, 4 patients). Nine patients $(56.2 \%)$ had symptoms with a duration of less than a week. There were 12 patients (75\%) who presented with visual acuity better than $6 / 60$, and 4 patients (25\%) presented with visual acuity worse than $6 / 60$. Out of 16 patients, acquired form of ocular toxoplasmosis was found in $75 \%$ (12 patients), and 25\% (4 patients) had a recurrent disease. No patient presented with congenital ocular toxoplasmosis. Patients with comorbidities, such as diabetes mellitus, remained low at $12.5 \%$. Interestingly, only $25 \%$ of the patients with ocular toxoplasmosis reported a history of owning a cat, but denied any history of cat scratches or bites.

The ocular manifestation of ocular toxoplasmosis at presentation is shown in Table 4 . Keratic precipitate $(75 \%)$ and vitritis (75\%) were the most common manifestation of ocular toxoplasmosis. Posterior synechiae comprised only $18.8 \%$ of the cases, with a Busacca nodule noted in only one of the patients. There were 8 patients $(50 \%)$ with retinitis at presentation, and out of these 8 patients, there were 4 patients with macular involvement, which eventually led to macular scarring. Both multifocal choroiditis and optic neuritis were reported in two patients (12.5\%). 


\begin{tabular}{|c|c|c|}
\hline Clinical profile & $\begin{array}{l}\text { Frequency } \\
\text { (n) }\end{array}$ & $\begin{array}{l}\text { Percentage } \\
(\%)\end{array}$ \\
\hline \multicolumn{3}{|l|}{ Laterality } \\
\hline Right eye & 9 & 56.3 \\
\hline Left eye & 4 & 25.0 \\
\hline Both eyes & 3 & 18.7 \\
\hline \multicolumn{3}{|l|}{ Presenting symptoms } \\
\hline Blurred vision & 12 & 75.0 \\
\hline Redness & 4 & 25.0 \\
\hline Floaters & 4 & 25.0 \\
\hline Eye pain & 3 & 18.8 \\
\hline \multicolumn{3}{|c|}{ Presenting Visual Acuity (Snellen chart) } \\
\hline Better than $6 / 60$ & 12 & 75.0 \\
\hline Worse than $6 / 60$ & 4 & 25.0 \\
\hline \multicolumn{3}{|l|}{ Course of disease } \\
\hline Congenital & 0 & 0.0 \\
\hline Acquired & 12 & 75.0 \\
\hline Recurrent & 4 & 25.0 \\
\hline \multicolumn{3}{|l|}{ Duration of symptoms } \\
\hline Less than 1 week & 9 & 56.2 \\
\hline Up to 1 month & 7 & 43.8 \\
\hline Diabetes mellitus & 2 & 12.5 \\
\hline Owns a Cat & 4 & 25.0 \\
\hline
\end{tabular}

\begin{tabular}{|c|c|c|}
\hline Ocular manifestation & \begin{tabular}{|l}
$\begin{array}{l}\text { Frequency } \\
(n)\end{array}$ \\
\end{tabular} & \begin{tabular}{|l}
$\begin{array}{l}\text { Percentage } \\
(\%)\end{array}$ \\
\end{tabular} \\
\hline Keratic precipitates & 12 & 75.0 \\
\hline Granulomatous & 4 & 33.3 \\
\hline Non-granulomatous & 8 & 66.7 \\
\hline Iris nodules & 1 & 6.2 \\
\hline Posterior synechiae & 3 & 18.8 \\
\hline Vitritis & 12 & 75.0 \\
\hline Retinitis & 8 & 50.0 \\
\hline Multifocal choroiditis & 2 & 12.5 \\
\hline Optic neuritis & 2 & 12.5 \\
\hline
\end{tabular}

ELISA was used qualitatively to determine the toxoplasmosis IgM and IgG to aid in the diagnosis of ocular toxoplasmosis after clinical features. Only 1 patient (6.7\%) was positive for IgM, while all patients were positive for IgG (except 1 missing data) (Table 5 ). Only 3 patients (20\%) showed an elevated erythrocyte sedimentation rate (ESR) of more than $25 \mathrm{~mm} /$ hour, and 1 patient (6.2\%) had an elevated total white blood cell count $\left(>11 \times 10^{9} / \mathrm{L}\right)$.

Patients diagnosed with ocular toxoplasmosis were treated with oral Azithromycin $500 \mathrm{mg}$ daily for a 6-week duration. Azithromycin was chosen as the primary antibiotic of choice, due to a higher compliance rate with the single daily administration dose and comparatively less side effects. Topical and oral steroids were administered according to the severity of ocular inflammation. A majority of patients (81.3\%, 13 patients) were treated with an instillation of steroid eye drops, while $25 \%$ (4 patients) required administration of an oral steroid. Oral Prednisolone at $1 \mathrm{mg} / \mathrm{kg}$ daily was started after commencement of treatment and was gradually tapered down within one month. Out of 16 patients, 3 patients required both steroid eye drops and oral steroids, due to severe ocular inflammation. There was no patient treated with subtenon or a subconjunctiva injection of steroids. In this case series, all patients were not treated with intravitreal Clindamycin injection. Upon completion of treatment, all 12 patients (75\%) presented with initial visual acuity better than $6 / 60$, which showed an improvement of vision. The remaining 4 (25\%) patients' visual acuity unfortunately did not improve better than $6 / 60$, despite completion of treatment, due to direct macular involvement. Patients treated successfully received a continued follow up at the Ophthalmology Clinic. As of a recent review, 4 of the patients (25\%) recorded having recurrent attacks, requiring re-treatment of Azithromycin and steroids. Out of 4 patients with recurrent attacks, 1 of them recorded having recurrent attacks of more than 5 separate episodes.

\begin{tabular}{|l|l|l|}
\hline \multicolumn{3}{|l|}{ Table 5. Laboratory profile of ocular toxoplasmosis } \\
\hline Laboratory profile & Frequency ( $n$ ) & Percentage (\%) \\
\hline Erythrocyte sedimentation rate (ESR) \\
\hline$<25 \mathrm{~mm} /$ hour & 12 & 80.0 \\
\hline$>25 \mathrm{~mm} /$ hour & 3 & 20.0 \\
\hline Missing data & 1 & - \\
\hline Toxoplasmosis immunoglobulin M (IgM) \\
\hline Positive & 1 & 6.7 \\
\hline Negative & 14 & 93.3 \\
\hline Missing data & 1 & - \\
\hline Toxoplasmosis immunoglobulin G (IgG) \\
\hline Positive & 16 & 100 \\
\hline Negative & 0 & 0 \\
\hline Total white cell count (x 10\%/L) & \\
\hline$<11$ & 15 & 93.8 \\
\hline$>11$ & 1 & 6.2 \\
\hline
\end{tabular}

\section{Discussion}

Ocular toxoplasmosis is one of the most common causes of ocular inflammation. A previous study by Suresh et al. [3] showed a $54.6 \%$ seroprevalence of $T$. gondii in the community of Kelantan, while Ngui et al. [4] documented a 37\% seroprevalence in the indigenous communities in Malaysia [3, 4]. This shows that the prevalence of toxoplasmosis infection is higher among the Kelantanese population than the indigenous communities in Malaysia.

Data analysis also demonstrated that more of the female population had ocular toxoplasmosis, which can be contributed to a higher seroprevalence among the female population. The higher rate of seroprevalence among the female population was echoed in a study by Ngui et al. [4], but there was no statistical significant difference between gender, suggesting that $T$. gondii infection was not gender related. This could point to the fact that activities performed by men and women among the community are different and have different exposures to risk factors, e.g. the female population is more involved in small-scale animal husbandry (e.g. chickens and ducks) and livestock rearing, whereas males are more involve in construction, agriculture or businesses-related occupations.

Although the youngest age that presented with ocular toxoplasmosis was 18 years old, toxoplasmosis infection could start at a much earlier age. Data from Ngui et al. [4] demonstrated a higher seroprevalence rate in participants aged above 12 years old. The source of infection would still most likely come from domestic felines, even though patients denied close contact, as a majority of the Kelantanese population are Muslims, and felines are among their favorite pets. Children may also acquire infection through unhygienic handling of food, poor knowledge of personal hygiene and washing of hands after handling or coming into contact with domestic felines. This could be aggravated by the lack of parental or adult supervision in personal hygiene, 
as both parents are usually working. The inability of children to provide more in-depth ocular complaints, such as reduced vision, could also be another reason children with ocular toxoplasmosis were not diagnosed earlier. The limitation of ocular inflammation and the self-limiting of red eye symptoms could often be dismissed for viral conjunctivitis, causing further delay in diagnosis and treatment.

In our review of the case series, we found that 7 out of 10 women presenting with ocular toxoplasmosis were of childbearing age. This shows a possibility of high-risk vertical transmission, therefore increasing the risk of congenital toxoplasmosis infection, which warrants the need for antenatal screening and screening of neonates in high-risk mothers in the local community.

The most common manifestation of ocular toxoplasmosis is vitritis (75\%), followed by retinitis (50\%). This is attributed to the nature of the inactive cyst, which remains in retina scars or nearby tissues. A rupture of the cyst releases bradyzoites into the surrounding tissues, triggering an immune response, such as vitritis and retinitis. Active lesions present as a whitish, fluffy lesion of the retina and adjacent to old hyperpigmented well-demarcated scar. Combination of whitish fluffy lesion with vitritis gives the typical "headlight in the fog" which is pathognomic of ocular toxoplasmosis. There were 4 patients $(25 \%)$ in the study population with retinitis involving the macular region. They presented with initial vision worse than $6 / 60$. Despite our best efforts and the treatment given, their visual acuity unfortunately remained the same. Patients without macular involvement were able to achieve near total visual recovery.

In our case series, the recurrence rate among patients with ocular toxoplasmosis was $25 \%$, in which 1 out of the 4 patients had a recurrence rate of more than 5 times. This is worrying, as patients presenting with a new infection are subject to recurrent ocular inflammation, which can lead to other morbidities.
Recurrent infection also warrants the possibility of a new retinal lesion and risk of significant vision morbidities if macular area is involved. Re-exposure to toxoplasmosis infection could be one of the causes for the recurrence, thus preventive measures and counselling are important in identifying the possible route of infection.

\section{Limitations and advantages of the study}

The limitation to this case series is that this is a retrospective review of cases of patients that presented to the institution with ocular toxoplasmosis over a period of 7 years. The diagnosis was made and treatment was provided by a different attending ophthalmologist at the time. Another limitation would be the number of cases which were not representative of the magnitude of toxoplasmosis infection nor the extent of ocular toxoplasmosis infection in the state or the country. However, this study does provide valuable insight into common ocular presentations from patients with ocular toxoplasmosis in this part of the world. The study also highlights the degree of severity, success of treatment, as well as the recurrence rate.

\section{Conclusions}

Ocular toxoplasmosis is a debilitating visual disease, which can significantly affect the activity of a patient in daily life and their function in society. Though causing severe ocular morbidity, toxoplasmosis infection can be prevented with preventive measures, such as habitual hand washing after contact with livestock, immunization of domestic pets, ensuring meat is properly cooked and a clean water source. Better awareness among the local community is also important in seeking treatment early, and identification of significant ocular inflammation is crucial to prevent further ocular damage.

Source of funding: This work was funded by the authors' own resources. Conflict of interest: The authors declare no conflict of interests.

\section{References}

1. Park YH, Nam HW. Clinical features and treatment of ocular toxoplasmosis. Korean J Parasitol 2013; 51(4): 393-399.

2. Bodaghi B, Touitou V, Fardeau C, et al. Toxoplasmosis: new challenges for an old disease. Eye 2012; 26(2): 241-244.

3. Suresh S, Nor-Masniwati S, Nor-Idahriani MN, et al. Serological IgG avidity test for ocular toxoplasmosis. Clin Ophthalmol 2012; 6: 147-150.

4. Ngui R, Lim YA, Amir NF, et al. Seroprevalence and source of toxoplasmosis among orang asli (indigenous) communities in penisular Malaysia. Am J Trop Med Hyg 2011; 85(4): 660-666.

5. Commodaro AG, Belfort RN, Rizzo LV, et al. Ocular Toxoplasmosis - an update and review of the literature. Mem Inst Oswaldo Cruz 2009; 104(2): 345-350.

6. Vasconcelos-Santos DV. Ocular manifestations of systemic disease: toxoplasmosis. Curr Opin Ophthalmol 2012; 23(6): 543-550.

7. Lim VK, Tan PL. Ocular toxoplasmosis in Malaysia. Med J Malaysia 1983; 38(3): 185-187.

8. Weiss LM, Dubey JP. Toxoplasmosis: a history of clinical observation. Int J Parasitol 2009; 39(8): 895-901.

9. Zierhut M, Deuter C, Murray PI. Classification of uveitis - current guidelines. European Ophthalmic Review 2007; 77-78, doi: http:// doi.org/10.17925/EOR.2007.00.00.77.

Tables: 5

Figures: 0

References: 9

Received: 14.10 .2017

Reviewed: 27.10.2017

Accepted: 06.12.2017

Address for correspondence:

Tong Jong Haw Matthew, MD

Department of Ophthalmology, School of Medical Sciences

Universiti Sains Malaysia

16150 Kubang Kerian

Kelantan, Malaysia

Tel: +6 097676362

Email:matthewtjh@gmail.com 